\title{
Magnetic complexity as an explanation for bimodal rotation populations among young stars
}

\author{
Cecilia Garraffo ${ }^{1}$, Jeremy J. Drake ${ }^{1}$, Ofer Cohen $^{1}$
}

\begin{abstract}
Observations of young open clusters have revealed a bimodal distribution of fast and slower rotation rates that has proven difficult to explain with predictive models of spin down that depend on rotation rates alone. The Metastable Dynamo Model proposed recently by Brown, employing a stochastic transition probability from slow to more rapid spin down regimes, appears to be more successful but lacks a physical basis for such duality. Using detailed 3D MHD wind models computed for idealized multipole magnetic fields, we show that surface magnetic field complexity can provide this basis. Both mass and angular momentum losses decline sharply with increasing field complexity. Combined with observation evidence for complex field morphologies in magnetically active stars, our results support a picture in which young, rapid rotators lose angular momentum in an inefficient way because of field complexity. During this slow spin-down phase, magnetic complexity is eroded, precipitating a rapid transition from weak to strong wind coupling.
\end{abstract}

Subject headings: stars: rotation — stars: magnetic field — stars: evolution

\section{INTRODUCTION}

Stellar rotation catalyzes magnetic dynamo activity in the interiors of late-type stars that is manifest at the surface in the form of magnetic fields, energetic photon and particle radiation, supersonic winds and coronal mass ejections. The magnetized winds carry away angular momentum, a process commonly referred to as "magnetic braking". As stars age, their rotation rates, $\Omega$, eventually converge to the empirical Skumanich (1972) spin down law $\Omega \sim t^{-1 / 2}$. Weber \& Davis (1967) and Mestel (1968) derived the first analytical expression for stellar angular momentum loss, obtaining $\dot{J}=\frac{2}{3} \Omega M R_{A}^{2}$, where $\dot{J}$ is the angular momentum loss rate, $\dot{M}$ the mass loss rate, $R_{A}$ is the radial distance at which the wind speed exceeds the local Alfvén speed (the "Alfvén radius"), and where a constant radial field was assumed at the surface of the star. This approach was later generalized to more realistic scenarios in a range of different studies (e.g. Mestel \& Spruit 1987; Kawaler 1988; Taam \& Spruit 1989, Chaboyer et al. 1995), which have generally proven successful in explaining spin down on the Main Sequence, including the empirical Skumanich law.

\footnotetext{
${ }^{1}$ Harvard-Smithsonian Center for Astrophysics, 60 Garden St. Cambridge, MA 02138
} 
The early phase of rotation evolution has proven more difficult to understand. Observations of young open clusters by (e.g. Stauffer \& Hartmann 1987; Soderblom et al. 1993; Queloz et al. 1998; Terndrup et al. 2000, see Meibom et al. 2011 for a recent compilation) found a large spread in rotation rates at ages up to a few hundred Myrs with a bimodal aspect comprising two branches corresponding to fast and slow rotation and implying an extremely fast transition between the two. The currently favored explanation for this phenomenon is a core-envelope decoupling near the zero age main-sequence (e.g. Stauffer et al. 1984; Soderblom et al. 1993; Barnes 2003), after which the outer convection zone with lower moment of inertia is rapidly spun down, leaving a more rapidlyrotating core. More recently, Brown (2014) has proposed a "Metastable Dynamo Model" (MDM) in which coupling between the magnetic field and wind is initially weak. Spontaneous strong coupling of the star to the wind then happens at a certain early age, initiating the rapid spin down. For $a d$ hoc coupling constant changes by factors of 100 or more, the model is successful in reproducing the observed rotation distributions of young clusters. However, the mechanism behind such a change in coupling has not been identified. Except for a small handful of indirect detections (e.g. Wood 2004; Wood et al. 2014), observational progress is stymied by the winds themselves being generally weak, while surface magnetic fields can only be inferred indirectly and with very limited spatial resolution (Donati \& Landstreet 2009).

One key ingredient in stellar rotation evolution models that has received scant attention is the morphology of the magnetic field. While the early treatments of Mestel \& Paris (1984) and Kawaler (1988) that provided much of the basis for subsequent rotation evolution models considered the multipole order of the magnetic field, this was limited to the effect of the radial dependence of the field strength. Moreover, spin down models have generally assumed dipolar fields. Instead, a growing database of Zeeman-Doppler imaging observations indicates that surface magnetic fields of young, active stars mainly consist of high-order multipole components, rather than a simple dipole such as characterizes the large-scale solar magnetic field (e.g. Donati 2003, Donati \& Landstreet 2009; Marsden et al. 2011; Waite et al. 2011, 2015). Linsky \& Wood (2014) have also recently inferred mass loss rates for the active stars $\xi$ Boo A and $\pi^{1}$ UMa that are two orders of magnitude lower than expected based on extrapolation from lower activity stars, suggesting that magnetic topology could have a more profound effect on angular momentum loss than simply through the radial field strength dependence.

Here, we investigate the role of magnetic field complexity on stellar angular momentum loss using a detailed, self-consistent three-dimensional magnetohydrodynamic (MHD) wind model that has proven successful in matching observations of the solar wind (Oran et al. 2013). We explore a range of simple magnetic configurations with different multipolar complexity. The numerical methods are described in Section 2, the results of model calculations in Section 3, and we discuss our main findings and their implications in Section 4. We conclude in Section 5 that magnetic complexity can provide the strong coupling switch sought in the MDM by Brown (2014). 


\section{NUMERICAL SIMULATION}

\subsection{MHD model}

In order to obtain solutions for the artificial stellar corona and wind cases considered here, we use the generic BATS-R-US code (Powell et al. 1999; Tóth et al. 2012) that solves the set of MHD equations for the conservation of mass, momentum, magnetic induction, and energy. A spherical grid, logarithmic in the $\hat{r}$ coordinate is used with adaptive mesh refinement to resolve current sheets and regions where the magnetic field changes sign.

The BATS-R-US module for the solar (or stellar) corona is driven by synoptic maps of the radial stellar magnetic field, which are used to specify the field boundary conditions. The initial condition for the three-dimensional field is obtained by calculating the potential field of these boundary conditions, assuming that the field is purely radial at a distance of $r=4.5 R_{\star}$ (the "source surface") (Altschuler \& Newkirk 1969). Typically, in the case of the Sun, the location of the source surface is closer in; we use this larger value to prevent the choice of this parameter from having an impact on the final solution. In the case of strong stellar magnetic fields, choosing a small value for the source surface can artificially truncate magnetic loops, resulting in an overpowering of the stellar wind to unrealistic values. This effect is eliminated as the source surface is moved outwards to larger distances, where it does not impact the MHD steady-state solution.

Once the initial condition for the magnetic field is specified, the model provides a self-consistent acceleration of the wind and heating of the corona via the Alfvén wave turbulence mechanism, including thermodynamic processes such as radiative cooling and electron heat conduction (see Oran et al. 2013; Sokolov et al. 2013; van der Holst et al. 2014, for full details). Unlike models that were used to study stellar winds with imposed, fully developed, spherically symmetric thermal ("Parker") winds (e.g., Matt et al. 2012; Vidotto et al. 2014b), here the wind and magnetic field solutions evolve together, allowing the magnetic field topology to influence the wind appropriately, as observed in the case of the solar wind in the heliosphere (Phillips et al. 1995; McComas et al. 2007).

\subsection{Simulations}

We perform three-dimensional stellar wind simulations for a hypothetical solar-mass star with a solar rotation period ( $\sim 25$ days) and for different magnetic topologies and field strengths. Our grid of fiducial magnetograms consists of the ten first magnetic moments for peak field flux densities of $B=10 \mathrm{G}, 20 \mathrm{G}$ and $100 \mathrm{G}$. The maps for different morphologies are built using the corresponding term in the multipolar expansion, i.e., a Legendre Polynomial times a phase. Examples of these fiducial magnetic maps are shown in Figure 1. For a constant peak field strength, the integrated magnetic flux declines slightly with increasing magnetic moment, $n$, according to the orthogonality

property $\int_{-1}^{1} P_{m}(x) P_{n}(x) d x=\frac{2}{2 n+1} \delta_{m n}$. For the $20 \mathrm{G}$ baseline case, we therefore also compute 
magnetograms normalized to the dipolar magnetic flux by the factor $\sqrt{2 /(2 \cdot 1+1)} / \sqrt{2 /(2 n+1)}=$ $\sqrt{(2 n+1) / 3}$.

From the three-dimensional model solutions we extract the wind density, $\rho$, and speed, u, over the Alfvén surface and at the stellar surface. The Alfvén surface itself is determined by finding the surface for which the wind speed reaches the local Alfvén speed, $v_{A}=B / \sqrt{\rho}$, neglecting the contribution of the electrons to the mass density, $\rho$, and for a pure hydrogen wind. We then compute the mass and angular momentum loss rates at each point of the Alfvén Surface, which in steady state, where all the gradients in pressure vanish, read:

$$
\begin{gathered}
\frac{d M}{d t}=\rho(\mathbf{u} \cdot \mathbf{d A}) \\
\frac{d J}{d t}=\Omega \rho R^{2} \sin ^{2} \theta(\mathbf{u} \cdot \mathbf{d} \mathbf{A}),
\end{gathered}
$$

where $\frac{d J}{d t}$ is the component of the angular momentum change in the direction of the rotation axis, and is the only one contributing to a change in the magnitude of $J$. Here, $\mathbf{d A}$ refers to the surface element on the Alfvén Surface. The formalism of Mestel (1999, see also the form used by Vidotto et al. (2014b), Eqn (A6)) includes an additional angular momentum term that accounts for net stresses in the system, which in steady state become zero.

We also calculate the amount of open magnetic flux through a spherical surface outside of the Alfvén surface.

\section{RESULTS}

The mass and angular momentum loss rates, together with amount of open flux computed for each case in our grid of models, are plotted in Figure 2.

The most dramatic result is a systematic decrease of both $\dot{M}$ and $\dot{J}$ by two and three orders of magnitude, respectively, with increasing complexity of the magnetic topology. For a given magnetic field strength, the logarithmic mass loss rate scales approximately linearly with magnetic moment, $\log \dot{M} \propto n$. Angular momentum loss is instead not a simple function of mass loss when including higher order magnetic moments but is very well correlated with the amount of open flux. The reasons underpinning these trends become clear when examining the details of the MHD model solutions and how the open field regions from which the wind is driven change with magnetic moment. The trends with constant magnetic flux are slightly less steep than for constant peak field, as expected from the general dependence of $\dot{M}$ and $\dot{J}$ on field strength for a given order of complexity.

There are three interrelated aspects to the angular momentum loss: the mass flux, the Alfvén radius over which it acts as a rotational brake, and the latitude at which the mass release happens. 
In order to compare the sizes and latitudes of the closed field line regions for the different magnetic morphologies, Figure 3 shows meridional cuts of the wind mass flux together with selected magnetic field lines for the $20 \mathrm{G}$ models. The area of the stellar surface occupied by open field decreases dramatically with increasing magnetic complexity, while open field regions ( "coronal holes") also get distributed more homogeneously over latitude. Only the first affects $\dot{M}$ directly, but both are expected to lead to a reduction of $\dot{J}$. The open field lines spread and overlay regions of closed field and the mass flux at the apex of the closed loop systems is larger than in the middle of open field regions. For the dipolar case this happens only at the equator, resulting in an equatoriallydominated mass loss, which is the most efficient latitude for losing angular momentum. This is also seen in Figure 5, where mass loss is plotted as a function of latitude for the $20 \mathrm{G}$ simulations. Increasing magnetic moment leads to a larger number of less pronounced local maxima. This change in latitudinal dependence of the mass loss leads to a stronger dependence of angular momentum loss on the magnetic complexity than just the mass loss rate itself (see Figure 2).

Solar wind observations show that the fast and less dense wind originates from the center of coronal holes, while the slow and denser solar wind originates from the boundary between the coronal holes and the closed loops (e.g. Phillips et al. 1995; McComas et al. 2007). Our results show that the size of the coronal holes decreases with the increase of the multipole order. As a result, the fast wind in the solution is eliminated to a point where only slow wind exists. On the other hand, the radial dependence of the magnetic field is proportional to $1 / r^{n+1}$, where $n$ is the magnetic multipole order. Therefore, the magnetic flux falls faster with radius for higher orders. As a consequence one should expect the Alfvén velocity at a given radial distance to decrease with magnetic complexity (much more than the wind speed), and the Alfvén radius to become smaller. Figure 4 shows how the three dimensional Alfvén surface of different magnetic morphologies (for the $20 \mathrm{G}$ models) shrinks rapidly with increasing complexity. The reduction in the wind speeds translates to a reduction of $\dot{M}$ and, therefore, $\dot{J}$, while the reduction in the lever arm due to the shrinkage of the Alfvén surface amplifies the reduction of $\dot{J}$ with higher magnetic multipole orders.

\section{DISCUSSION}

Brown (2014) has discussed in detail the difficulties existing stellar spin down models have in matching the observed distributions of stellar rotation velocities in young open clusters. Coreenvelope decoupling models are able to provide a reasonable match with specified percentile points in the distribution of rotation periods, $P_{r o t}$, but fail in reproducing its bimodal aspect and how it changes with time.

Barnes (2003) dubbed the bimodal branches $C$ for the rapid rotators and $I$ for the slower ones, tentatively identifying these as being dominated by convective and interface dynamos, respectively. He speculated that on the $C$ sequence, the magnetic coupling of the star to the wind was weak, possibly due to the magnetic field morphology. Barnes \& Kim (2010) fleshed out the ideas of Barnes (2003) and the core-envelope decoupling idea, synthesizing a purely descriptive expression 
for period evolution from the two different regimes that Brown (2014) referred to as the Symmetrical Empirical Model (SEM). While the SEM appears to be able to match the general distribution of periods seen in open clusters, Brown (2014) concludes that, as specified, it also fails to match the details of the bimodal morphology, and in particular in being able to sustain $\mathrm{G}$ stars with ages above $200 \mathrm{Myr}$ and $P_{\text {rot }} \leq 2$ days, as are observed in M34 and M37.

Brown (2014) emphasizes that existing models might be improved to better reproduce observations, but for simplicity introduced a different concept that amounts to an ad hoc mass-dependent transition probability between the $C$ and $I$ states. The MDM, briefly introduced in Section 1 . posits that stars are born with their magnetic dynamos operating in a mode that couples very weakly to the stellar wind and, at some point, this mode spontaneously and randomly changes to a strongly-coupled mode. Stars then spin down following the torque law,

$$
\frac{d J}{d t}=K_{M} \Omega^{3} f^{2}(B-V),
$$

where, as in the SEM model (Barnes \& Kim 2010), $B-V$ stands for color and $f^{2}(B-V)$ is a function that depends on stellar parameters. The constant $K_{M}$ may take two values corresponding to the strong and the weak-coupling regimes, the latter smaller than the former by a factor of 100 or more. One major difference between the MDM concept and other models is that the introduction of a random transition between the different modes means that the early evolution of $P_{\text {rot }}$ for a given star is not a simple deterministic function of its initial rotation rate. While faring less well in matching the details of the $I$ sequence, the MDM model appears successful in qualitatively matching the bimodal structure of $P_{\text {rot }}$ distributions at young ages. What the model lacks is a physical mechanism responsible for the coupling change.

Our MHD simulations indicate that the efficiency of angular momentum loss is strongly suppressed for stars with complex surface magnetic fields. The quadrupolar case already represents almost an order of magnitude decline compared with a dipolar field, irrespective of the absolute field strength, while for high order fields both mass and angular momentum losses are almost independent of the magnetic field strength. The results have a conspicuous parallel to the coupling constant proposed by Brown (2014), and indicate that, as has already been mentioned qualitatively in the past (e.g. Taam \& Spruit 1989; Barnes 2003; Vidotto et al. 2014a), magnetic complexity could be a key missing factor required to understand stellar rotation. In the light of growing evidence pointing to a systematic increase of higher-order magnetic moments in younger stars, we propose that the evolution of magnetic morphology on the stellar surface (determined by the evolution of the stellar dynamo) provides a simple explanation for the quite different $\dot{J}$ regimes that appear to be required to match observations of early stellar rotation evolution.

If we identify $K_{M}$ with magnetic complexity, for a solar-like star with a constant $\Omega$ we find that the change in $K_{M}$ induced by a change of morphology (dipolar vs multipolar cases) based on Figure 2 can be

$$
\frac{\frac{d J}{d t} d i p}{\frac{d J}{d t} \text { mult }}=\frac{K_{M_{1}}}{K_{M_{0}}} \sim 250,
$$


which is sufficient for the requirements of the MDM (Brown 2014). The case $K_{M_{0}}$, referring to the regime in which the star is effectively decoupled from its wind, then corresponds to our multipolar topology, while $K_{M_{1}}$, referring to strong coupling, corresponds to development of a strong dipolar component. In Brown's approach the transition probability for this flip is mass dependent and scales with the turnover time $\tau\left(M_{\star}\right)$. In our framework, this is equivalent to a mass dependent timescale for the evolution of the dynamo and magnetic morphology.

The results presented here show that magnetic complexity can provide almost an "off-on" coupling switch between a star and its wind. This results from a combination of suppressed mass loss, a steeper dependence of magnetic field with radial distance and more compact Alfvén surface, and the shift of the dominant mass loss from equatorial in the dipolar case to more sphericallysymmetric as complexity increases. The MDM assumes an instantaneous irreversible transition between dynamo modes. In reality, there could be a period of flip-flopping between different states of complexity, perhaps combined with a more gradual transition from higher order to lower order states. There is, however, currently no solid theoretical basis for an underlying dynamo change at rotation periods of a few days that might give rise to such changes. The scenario could be tested observationally through systematic examination of surface magnetic field maps of stars on the $C$ and $I$ sequences, extending the existing sample for stars on the $C$ sequence, and measurement of their wind-driven mass loss rates. While the apparently lower mass loss rates found for $\xi$ Boo A and $\pi^{1}$ UMa by Linsky \& Wood (2014) are encouraging, with rotation periods of 6.3 and 5 days, respectively (Donahue et al. 1996, Maldonado et al. 2010) these stars have probably transitioned recently to the $I$ sequence.

\section{CONCLUSIONS}

Using detailed 3D MHD models of a solar-like wind, we find that mass loss and spin down rates get rapidly suppressed with increasing complexity of the stellar magnetic field. Higher order magnetic moments generate a magnetosphere with a larger number of closed magnetic field lines that suppress mass loss. For higher order magnetic moments, the spin-down rate is no longer a simple function of the mass loss rate. Both closed and open field line regions become more homogeneously distributed over latitude for increasing magnetic complexity. As a consequence, mass loss is no longer equatorially dominated as for the dipolar case, and angular momentum loss is less efficient. Furthermore, the steeper radial decrease of the magnetic field magnitude for higher order multipoles results in a smaller Alfvén surface and a shorter lever arm for magnetic braking.

Echoing the MDM of Brown (2014), we propose a new interpretation for the bimodal rotation distribution observed in young clusters: Young solar-like stars have complex magnetic morphologies and lose angular momentum in an inefficient way. During this slow spin-down, magnetic complexity is eroded, precipitating a rapid transition from weak to strong wind coupling. The very rapid and drastic transition to the efficient spin down regime is caused by the very steep dependence of angular momentum loss on magnetic complexity found in this study. 
CG and OC were supported by SI Grand Challenges grant "Lessons from Mars: Are Habitable Atmospheres on Planets around M Dwarfs Viable?". OC was also supported by SI CGPS grant "Can Exoplanets Around Red Dwarfs Maintain Habitable Atmospheres?". JJD was supported by NASA contract NAS8-03060 to the Chandra X-ray Center. Numerical simulations were performed on the NASA HEC Pleiades system under award SMD-13-4526.

\section{REFERENCES}

Altschuler, M. D., \& Newkirk, G. 1969, Sol. Phys., 9, 131

Barnes, S. A. 2003, ApJ, 586, 464

Barnes, S. A., \& Kim, Y.-C. 2010, ApJ, 721, 675

Brown, T. M. 2014, ArXiv e-prints

Chaboyer, B., Demarque, P., \& Pinsonneault, M. H. 1995, ApJ, 441, 865

Donahue, R. A., Saar, S. H., \& Baliunas, S. L. 1996, ApJ, 466, 384

Donati, J.-F. 2003, in EAS Publications Series, Vol. 9, EAS Publications Series, ed. J. Arnaud \& N. Meunier, 169

Donati, J.-F., \& Landstreet, J. D. 2009, ARA\&A, 47, 333

Kawaler, S. D. 1988, ApJ, 333, 236

Linsky, J. L., \& Wood, B. E. 2014, ASTRA Proceedings, 1, 43

Maldonado, J., Martínez-Arnáiz, R. M., Eiroa, C., Montes, D., \& Montesinos, B. 2010, A\&A, 521, A12

Marsden, S. C., Jardine, M. M., Ramírez Vélez, J. C., Alecian, E., Brown, C. J., Carter, B. D., Donati, J.-F., Dunstone, N., Hart, R., Semel, M., \& Waite, I. A. 2011, MNRAS, 413, 1922

Matt, S. P., MacGregor, K. B., Pinsonneault, M. H., \& Greene, T. P. 2012, ApJ, 754, L26

McComas, D. J., Velli, M., Lewis, W. S., Acton, L. W., Balat-Pichelin, M., Bothmer, V., Dirling, R. B., Feldman, W. C., Gloeckler, G., Habbal, S. R., Hassler, D. M., Mann, I., Matthaeus, W. H., McNutt, R. L., Mewaldt, R. A., Murphy, N., Ofman, L., Sittler, E. C., Smith, C. W., \& Zurbuchen, T. H. 2007, Reviews of Geophysics, 45, 1004

Meibom, S., Mathieu, R. D., Stassun, K. G., Liebesny, P., \& Saar, S. H. 2011, ApJ, 733, 115

Mestel, L. 1968, MNRAS, 138, 359

—. 1999, Stellar magnetism, Oxford University Press, New York, USA 
Mestel, L., \& Paris, R. B. 1984, A\&A, 136, 98

Mestel, L., \& Spruit, H. C. 1987, MNRAS, 226, 57

Oran, R., van der Holst, B., Landi, E., Jin, M., Sokolov, I. V., \& Gombosi, T. I. 2013, ApJ, 778, 176

Phillips, J. L., Bame, S. J., Barnes, A., Barraclough, B. L., Feldman, W. C., Goldstein, B. E., Gosling, J. T., Hoogeveen, G. W., McComas, D. J., Neugebauer, M., \& Suess, S. T. 1995, Geophys. Res. Lett., 22, 3301

Powell, K. G., Roe, P. L., Linde, T. J., Gombosi, T. I., \& Zeeuw, D. L. D. 1999, Journal of Computational Physics, 154, 284

Queloz, D., Allain, S., Mermilliod, J.-C., Bouvier, J., \& Mayor, M. 1998, A\&A, 335, 183

Skumanich, A. 1972, ApJ, 171, 565

Soderblom, D. R., Stauffer, J. R., MacGregor, K. B., \& Jones, B. F. 1993, ApJ, 409, 624

Sokolov, I. V., van der Holst, B., Oran, R., Downs, C., Roussev, I. I., Jin, M., Manchester, IV, W. B., Evans, R. M., \& Gombosi, T. I. 2013, ApJ, 764, 23

Stauffer, J. R., Hartmann, L., Soderblom, D. R., \& Burnham, N. 1984, ApJ, 280, 202

Stauffer, J. R., \& Hartmann, L. W. 1987, ApJ, 318, 337

Taam, R. E., \& Spruit, H. C. 1989, ApJ, 345, 972

Terndrup, D. M., Stauffer, J. R., Pinsonneault, M. H., Sills, A., Yuan, Y., Jones, B. F., Fischer, D., \& Krishnamurthi, A. 2000, AJ, 119, 1303

Tóth, G., van der Holst, B., Sokolov, I. V., De Zeeuw, D. L., Gombosi, T. I., Fang, F., Manchester, W. B., Meng, X., Najib, D., Powell, K. G., Stout, Q. F., Glocer, A., Ma, Y.-J., \& Opher, M. 2012, Journal of Computational Physics, 231, 870

van der Holst, B., Sokolov, I. V., Meng, X., Jin, M., Manchester, IV, W. B., Tóth, G., \& Gombosi, T. I. 2014, ApJ, 782, 81

Vidotto, A. A., Gregory, S. G., Jardine, M., Donati, J. F., Petit, P., Morin, J., Folsom, C. P., Bouvier, J., Cameron, A. C., Hussain, G., Marsden, S., Waite, I. A., Fares, R., Jeffers, S., \& do Nascimento, J. D. 2014a, MNRAS, 441, 2361

Vidotto, A. A., Jardine, M., Morin, J., Donati, J. F., Opher, M., \& Gombosi, T. I. 2014b, MNRAS, 438, 1162

Waite, I. A., Marsden, S. C., Carter, B. D., Hart, R., Donati, J.-F., Ramírez Vélez, J. C., Semel, M., \& Dunstone, N. 2011, MNRAS, 413, 1949 
Waite, I. A., Marsden, S. C., Carter, B. D., Petit, P., Donati, J.-F., Jeffers, S. V., \& Boro Saikia, S. 2015, MNRAS, 449, 8

Weber, E. J., \& Davis, Jr., L. 1967, ApJ, 148, 217

Wood, B. E. 2004, Living Reviews in Solar Physics, 1, 2

Wood, B. E., Müller, H.-R., Redfield, S., \& Edelman, E. 2014, ApJ, 781, L33 

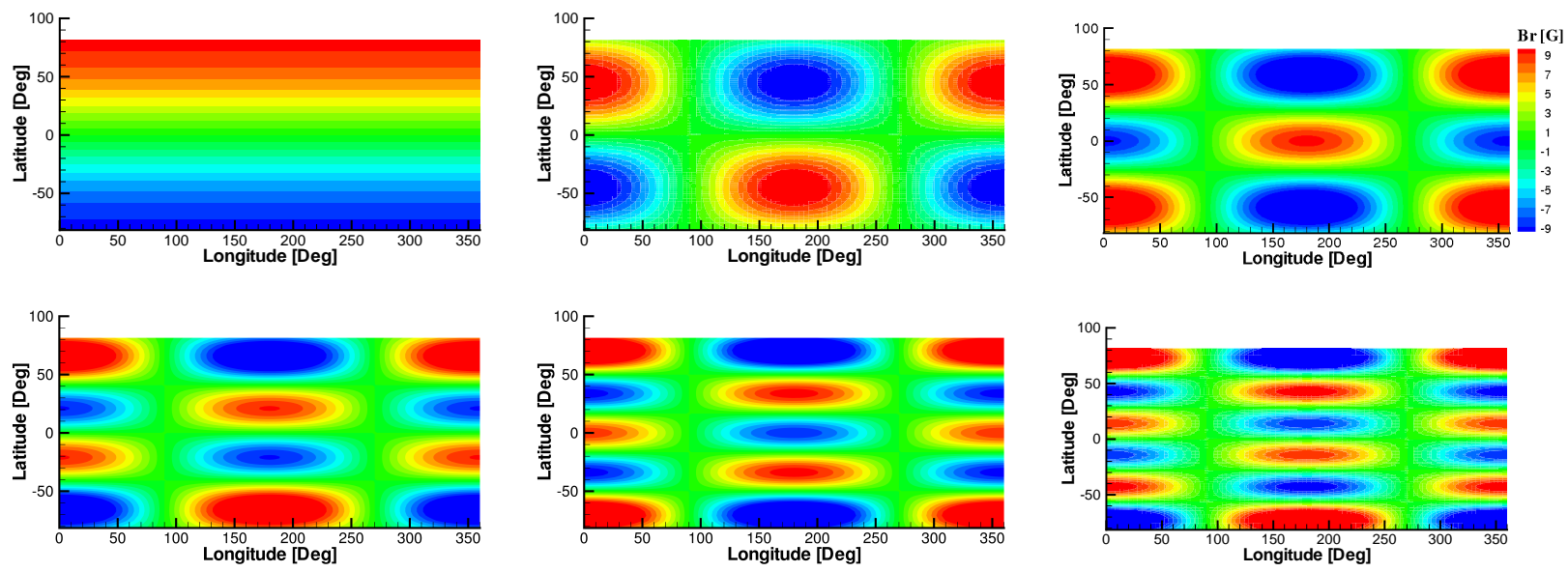

Fig. 1.- Illustration of representative fiducial magnetograms for magnetic flux densities of increasing magnetic multipole orders (from top left to right bottom) up to order 6 for the $10 \mathrm{G}$ amplitude case. Those for $20 \mathrm{G}$ and $100 \mathrm{G}$ cases have the same appearance with rescaled amplitude. 

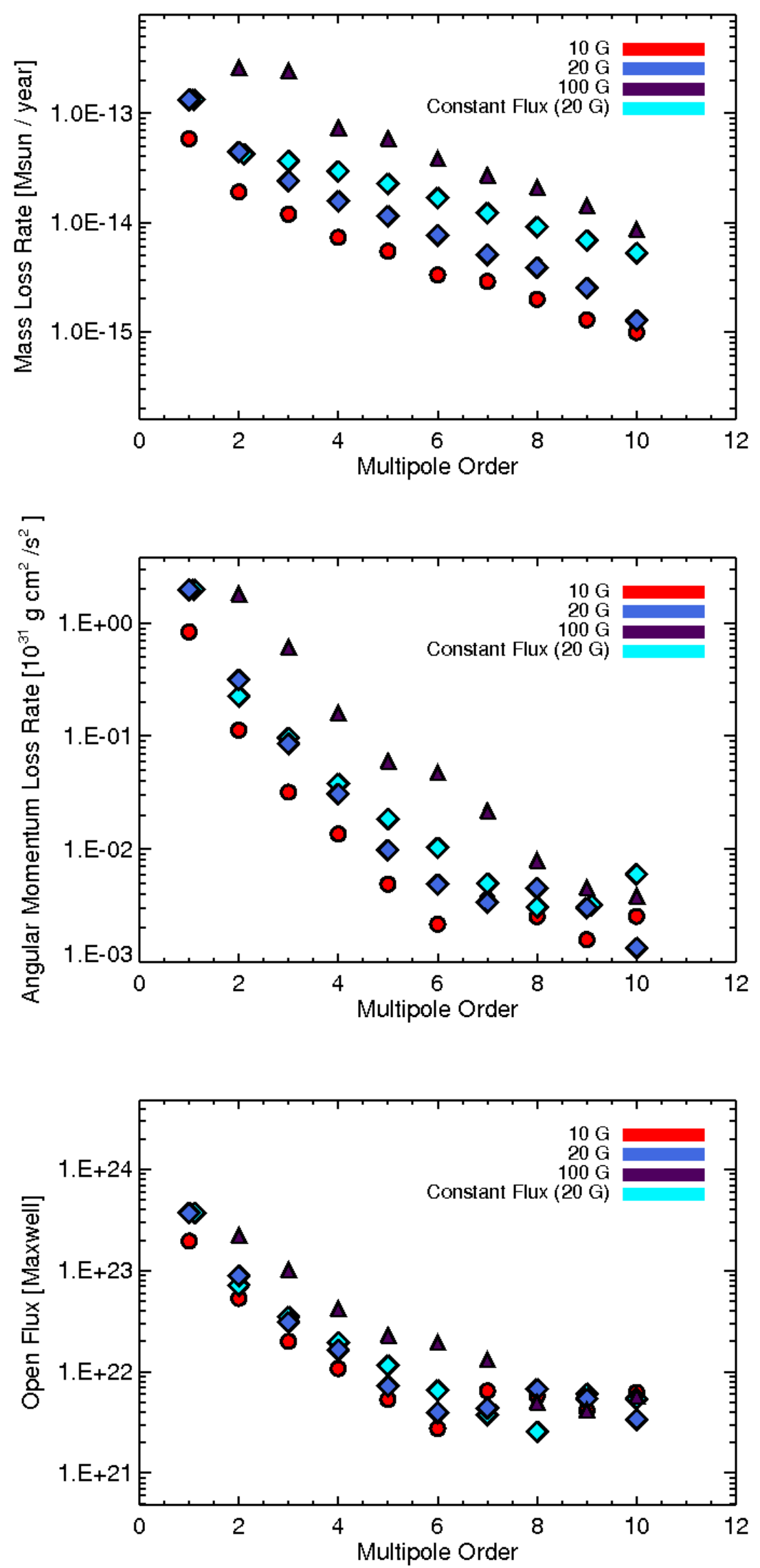

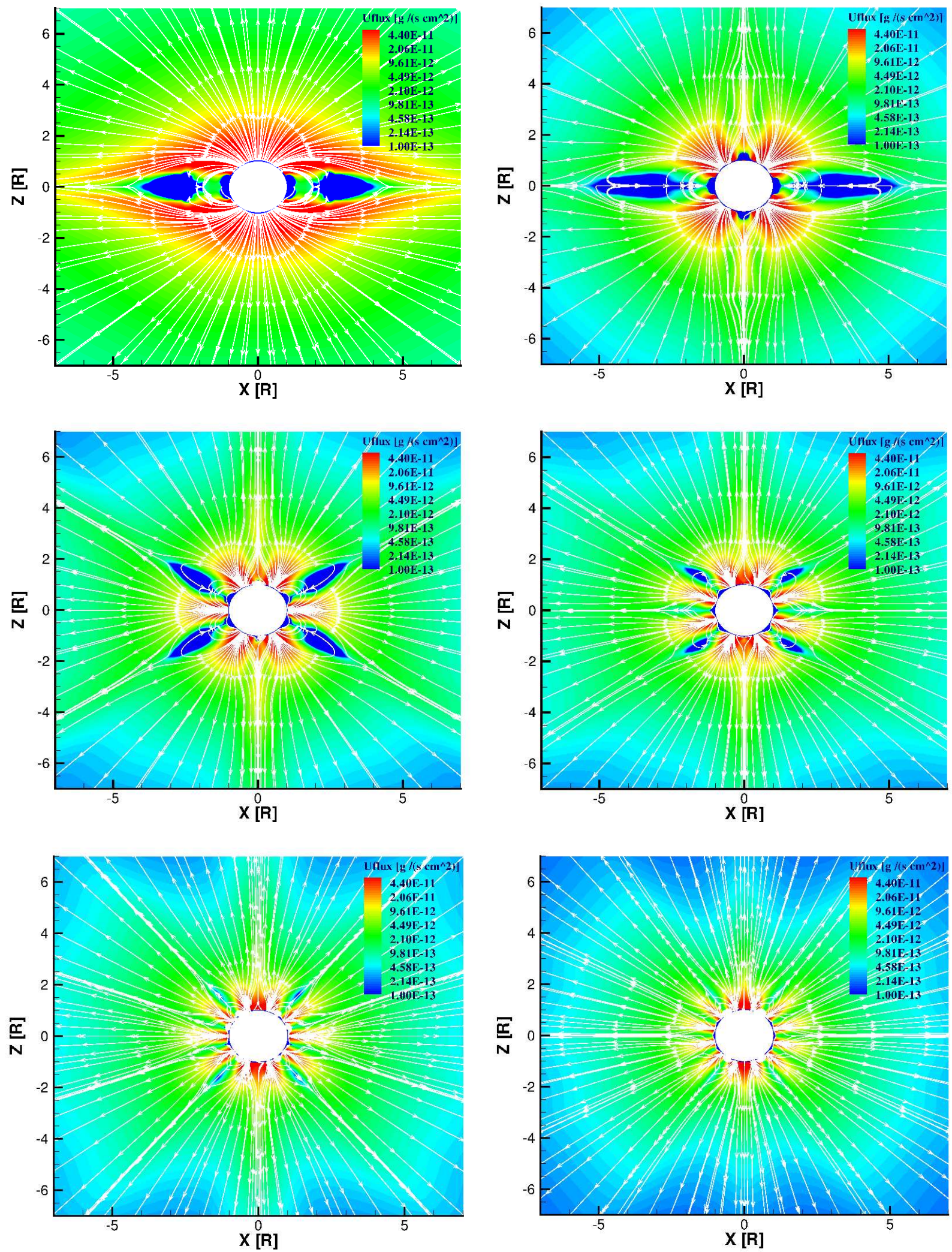

Fig. 3. - Meridional cut of the wind mass flux for increasing magnetic complexity for $20 \mathrm{G}$ models, from a dipole (top left) to a 6th order multipole (bottom right). Magnetic field lines are plotted in white and axis units are in stellar radii. 

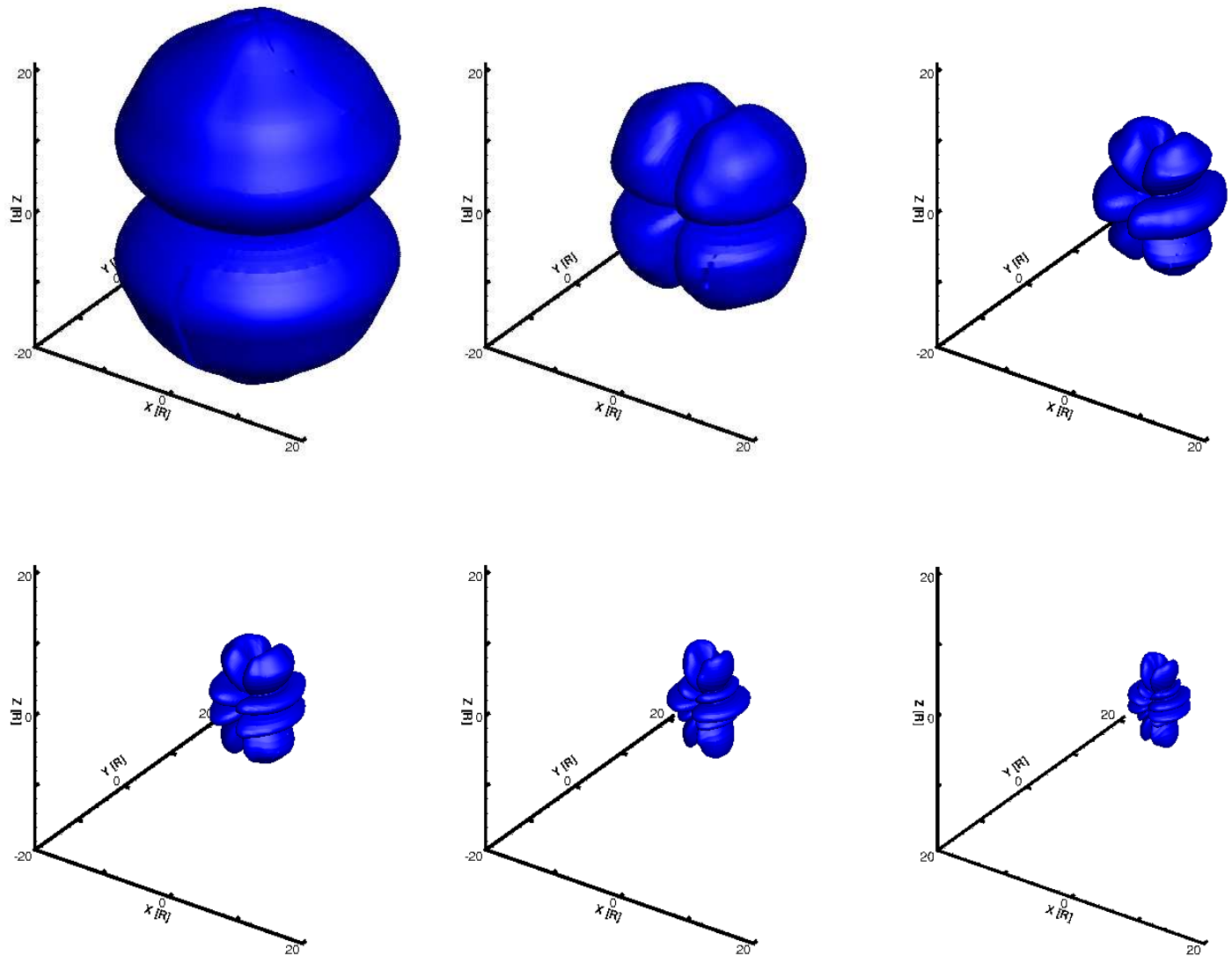

Fig. 4.- Three dimensional Alfvén surfaces for $20 \mathrm{G}$ fields of increasing complexity, from a dipole (top left) to a 6 th order magnetic multipole (bottom right). 


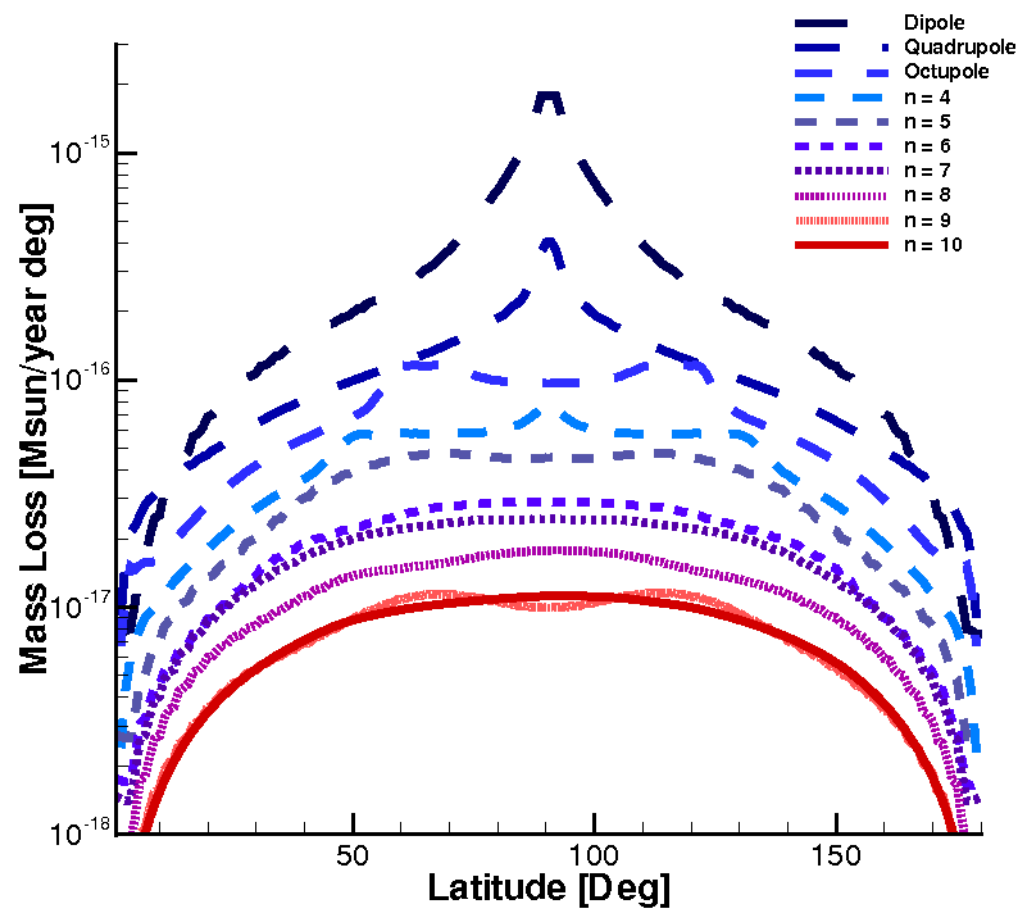

Fig. 5.- Mass loss distribution in latitude for different morphologies of $20 \mathrm{G}$ magnetic field strength, where $n$ refers to magnetic multipole order. 